\title{
Language across the mind and brain
}

\section{Manuel Carreiras*}

Basque Center on Cognition, Brain and Language, IKERBASQUE, Basque Foundation for Science, San Sebastian, Spain

*Correspondence: m.carreiras@bcbl.eu

Understanding how language works is a complex enterprise. The last 40 years have been witness to a remarkable effort from several disciplines including, experimental psychology, neuroscience, linguistics, computer science, etc. to uncover the cognitive and brain mechanisms underlying language acquisition, comprehension and production. The study of language processing has blossomed in the past two decades. Much of this expansion in research and interest has been driven by the confluence of fertile theoretical perspectives, refined experimental tasks, and by newer and better neuroimaging techniques of investigation. The focus of psycholinguistics has traditionally been the study of normal and impaired individuals using behavioral methods such as error rates, reaction time measures and, more recently, eye tracking. Important theoretical and experimental progress has been achieved by characterizing the types of cognitive representations and operations that underlie language processing. These advances have been based on how language is acquired, comprehended and produced under restricted conditions in the laboratory, as well as how it breaks down after focal brain lesions or during neurodegeneration. The information-processing paradigm underlying cognitive psychology led to new insights by combining laboratory data with information about the selective deficits of brain damaged patients. The fact that specific cognitive functions can be severely impaired while others remain intact in the same person is precious information to understand how the machine works. The characterization of language disorders that result from brain damage has had important implications both for theories of the cognitive processes underlying language and for theories of how language is represented and processed in the brain.

More recently, the interest in relating cognitive function to human brain activity has further advanced with the advent of highly sophisticated non-invasive brain imaging methods such as electroencephalography (EEG), magnetoencephalography (MEG), functional magnetic resonance imaging ( $\mathrm{fMRI}$ ), diffusion tensor imaging (DTI), etc. These new methods opened new avenues for understanding how our brains process language, because they made it possible to observe brain changes with high temporal and/or spatial resolution in intact brains of volunteers while they carry out experimental tasks. Imaging techniques provide a unique opportunity to assess biological and behavioral changes simultaneously and therefore for establishing brain-behavior associations. The fact that the new neuroimaging methods can be used with healthy volunteers is very important, since it allows combining the methods in intelligent ways with previous knowledge of tasks, procedures and paradigms from experimental psychology. The careful design of tasks, procedures and paradigms to investigate different components of language processes is absolutely critical if we want to make use of the data regardless of the specific neuroimaging recording technique, including the most sophisticated.

Apart from classical cognitive psychology and the new neuroimaging methods, a third important approach for understanding language involves computational modeling of cognitive functions. Connectionist models emerged 20 years ago, shifting the classical analogy of the mind as a serial information processor (computer) toward an analogy claiming an abstract similarity between artificial computational models and neural populations. This shift also returned learning to the focus of attention. Computational models in different areas (e.g., visual word recognition, auditory word recognition, semantic memory) helped to formalize the current knowledge of particular areas and to generate predictions that could be compared to human data. They were a step in the direction toward brain-style computation. It is also very important to mention the large body of computational linguistics work based on data driven distributional approaches with a more engineering oriented approach.

The cognitive plausibility of these formal models has primarily been evaluated against grammaticality judgments, semantic feature norms, errors, reaction times, etc. These formal perspectives are beginning to have more plausible neural perspectives, and recent computational models try to incorporate more neural detail. Computational neuroscience of language is an emerging research area that integrates recent advances in computational modeling and cognitive neuroscience, with the objective of developing cognitively and neurally plausible models of language.

The three avenues tend to merge in the highly interdisciplinary field of Cognitive Neuroscience. Nowadays, most cuttingedge research on language processing drinks from these three fountains. The progress has been remarkable in the last few years, with an increasing number of new empirical findings, theoretical advances and methodological breakthroughs. Nonetheless, despite all the advances in the last decades our brain is still a black box and our understanding of how language works is still scarce. We need to visualize the cognitive and brain circuits of language comprehension and production: how the brain computes in fractions of seconds an information flow from sounds or letters to meanings and from meanings to sounds or letters; how specific language impairments are tightly related to specific damage in some parts of the circuits; what the link is between some genetic mutations, brain malformations and language disorders. No other machine is so precious and yet so poorly understood as the brain and its most human unique ability: language. Like most problems in science, the answers that studies of language in cognition and in the human brain yield provoke only further, more interesting questions.

The journal Frontiers in Language Sciences aims to be a forum to discuss new answers and new questions and host future exciting developments in the field. The 
coming years will face at least three streams of challenges: understanding the computational mechanisms of language processing, language acquisition and language learning; the linking between brain and cognition so that new cognitive models have brain plausibility; and how cognitive neuroscience can inform other areas such as education and rehabilitation.

A distinctive feature of the human brain is its capacity to learn and adapt to an everchanging environment. The process of language acquisition and language learning is still a mystery. What sort of cognitive mechanisms and brain plasticity allow and promote these processes? We are still struggling with how two languages are processed in one brain, and what factors are responsible for producing such a variety of answers to this complex question. Is there any way to settle endless debates about what is the contribution of statistical learning, age of acquisition or proficiency? Similar endless debates regarding the cognitive architecture of production and comprehension processes are taking place. Are such debates, which mimic each other (modularity vs. interactivity) at different levels of processing still useful? Do we need to approach them from a different perspective? The impact of universal and language specific features of languages on processing, the effects of modalities (sign language vs. speech; speech vs. reading) and the relation of language with other cognitive abilities will help to understand language itself.

Timing is a very important property for information processing as it is for transfer of information among brain regions. Classical studies of information processing and representation are needed to understand how we compute linguistic information at different levels, but fresh theoretical views need to be developed to go beyond the debate about cognitive architecture (modular vs. interactive). A better understanding of the physiological interaction between different cortical regions of a particular network involved in a particular linguistic task may help us to constrain how processing at the cognitive level is taking place. The sequencing of the genome can be relatively easily done nowadays with current technology. However, some years ago it was a herculean task. It is possible to imagine that something similar may happen with language processing, by using the classical experimental procedures together with the new neuroimaging technologies guided by good theoretical frameworks for elaborating maps of the circuits of the human brain. Understanding how language works in the healthy and impaired brain will entail the study of the functional and structural neuroanatomy of the language circuits, the use of complex simulations, and a more refined theoretical framework relating different levels of explanation to crack the neural code, ultimately uncovering how the electric impulses are translated intro representations and sounds. Crossing different levels of explanation linked to cognitive function is going to be crucial for this enterprise. There is new work relating, for instance, molecular variations at the genetic level with behavior, with structural changes in the brain and with neural activity in particular brain circuits. Another important move is to go beyond the role of each particular brain area and its possible contribution to a particular language function to understand how brain circuits work and sustain such function, that is, how various regions interact in neural networks. Other avenues for gaining a better understanding of the human language system include decoding cognitive states from recordings of neural activity of very simple language changes or categories, or linking the dynamics of learning with changes in brain plasticity. The ultimate goal would be to link patterns of activation of several cells and the dynamics of these patterns of activation with mental states, such as representations of words or sentences, etc. Thus, we need to link structural and functional brain connectivity changes with learning, as when a person learns a new language. DTI fractional anisotropy is a methodological tool allowing steps in this direction, even though it is an indirect correlate of myelination or structural integrity. During development or during learning, structural changes presumably reflect ongoing myelination of axons, which enhances neuronal conduction and may play a role in the speed of cognitive processing. Understanding the development of pathways underlying language learning and language processing and the experiences that alter those pathways is imperative.

Education and rehabilitation are among the areas that may benefit from new findings in Cognitive Neuroscience. For instance, as learning and education are closely related to the processes of brain development, examining typical and atypical development has the potential to inform the educational process. Regarding language, particularly promising is the early identification of risk for specific language impairment and/or dyslexia, the identification of why some children learn to read whereas others fail in mastering such a basic skill. This research may clarify why some children with reading problems respond to intervention while others do not, which is essential in order to improve intervention programs to avoid language and/ or reading failure. Rehabilitation therapies from strokes or during neurodegenerative processes can also be improved with a better understanding of how language works, which at the same time will benefit from understanding what brain and cognitive mechanisms fail in these situations.

In sum, Frontiers in Language Sciences aims to host new findings related to the challenge of understanding how language, a specific human ability, works in the mind and in the brain.

Received:06 April 2010; accepted: 13 April 2010; published online: 07 May 2010.

Citation: Carreiras M (2010) Language across the mind and brain. Front. Psychology 1:14. doi: 10.3389/ fpsyg.2010.00014

This article was submitted to Frontiers in Language Sciences, a specialty of Frontiers in Psychology.

Copyright (@) 2010 Carreiras. This is an open-access article subject to an exclusive license agreement between the authors and the Frontiers Research Foundation, which permits unrestricted use, distribution, and reproduction in any medium, provided the original authors and source are credited. 